\title{
Spinal Arteriovenous Malformation in the Cervical Area (A Case report with brief review of literature)
}

\author{
TALIB S.H*, Pole S**, Sainani Rahul ***, Deshpande Shreyas***, \\ Dr Korde Krishna**** \\ * Professor \& Head, **Interventional Radiologist, ***Chief Resident \\ ****Senior Resident Department Of Medicine \& Interventional Radiology Department, Mahatma Gandhi \\ Missions Medical College \& Hospital , Aurangabad.431003
}

\begin{abstract}
Cranio cervical junction intra medullary subdural AVF/AVM (type $2 A V M$ ) is a rare \& enigmatic disease. We present a case of 25 yrs old male patient of cervical spinal, intramedullary arterio venous malformation with diffuse type nidus presented initially as bladder retention \& myelo pathic changes at a later date . Endovascular occlusion using glue adhesive embolisation was undertaken. Post embolisation angiography documented complete obstruction of AVM with good subtle recovery without any procedural related complications. Diagnosing these lesions early \& providing appropriate treatment is important if patients are to achieve an optimal neurologic outcome.
\end{abstract}

\section{Introduction :}

Dural arteriovenous fistulas ( DAVFS ) or AVM are classified according to their location, drainage pattern \& early venous drainage. ${ }^{1,2}$ The venous drainage pattern is considered a vital predictor of clinical behaviour . Presentations could either be with haemorrhage, venous infarct or myelopathy . The recognition of vascular malformations of the spinal cord comprising 3-4 \% of spinal masses are important as the clinical entities may yield devastating severe neurological deficits or even death if entity not recognized timely. Due to rare occurrence the lesions are seldomly considered in the differential diagnosis of progressive myelopathy. We describe a patient of type 2 AVM of cervical cord region recently seen \& treated with endovascular embolisation. The article deals with, clinical presentation, case evaluation \& brief review of different types of spinal AVMs with their clinical significance and management.

\section{Clinical case presentation :}

SV , 25 yrs right handed male patient was seen by urologist for retention of urinary bladder 9 months prior to the present hospitalization. Investigative cystoscopy with ascending cystourethrography was inconclusive. On catheter removal he developed urinary \& bowel incontinence . 3 months later he developed right lower limb weakness . 10 days prior to the present hospitalisation weakness was noticed in left lower limb with difficulties in getting up from lying down position. By the time, neuro evaluation the patient required assistance for ambulation. The weakness was symmetric in both lower extremities with proximal \& distal musculature affected equally. He also experienced bilateral lower extremity tingling . There was no significant associated pains. He did not offer history of either cranial or spinal trauma.

Examination revealed diminished motor strength $1 / 5$ bilaterally in lower limbs with distal musculature affected more than proximal . Patient noted to have diffuse, decrease in sensitivity to pin prick, cold $\&$ hot sensations with normal light touch in lower limbs. Proprioception \& vibratory sense were normal. Muscle wasting in deltoid \& forearm on either sides were observed. The deep tendon reflexes were hyper active 3/4 in upper \& 4/4 in lower extremities with patellar \& ankle clonus. Babinskis reflexes were present bilaterally . Abdominal reflexes were absent . Rectal examination revealed poor tone. Cremasteric were absent bilaterally . There were no fasciculations.Clinical diagnosis of ? cervical syringomyelia / non compressive cervical myelopathy was entertained. The patient was evaluated with contrast MRI of cervical, thoracic \& lumbar spine . The cord was enlarged in cervical region C2-C7 with hyperintense signals . Serpinginous multiple signals void areas noted with enhancement on contrast suggested possibility of spinal dural AVM/fistula ( Fig 1 ). Cerebral \& cervical spine angiography ( DSA ) revealed single feeder from the left vertebral artery \& signs of arterio venous malformation ( Fig 2,3 ) .Endovascular glue embolisation was carried out . Post embolisation angiography documented complete obliteration without any procedural related complications ( Fig 4 ). Effective outcome was noted. Post procedure 3 weeks later, patient had shown remarkable improvement over bladder \& anal control, improvement in sensations, disappearance of clonus \& improvement in power in both lower extremities. He is capable of getting up from lying position without assistance. He is presently undergoing physiotherapy for rehabilitation of the extremity weakness. 


\section{Discussion :}

Arterial \& venous spinal cord vascular malformations represent a heterogenous group of blood vessel disorders that affect the spinal cord parenchyma either directly or indirectly. These lesions include arterivenous malformations ( AVMS ), duro arteriovenous fistula ( DAVF ), spinal haemangiomas \& aneurysms. AVMS \& AVFS recognitions are important as these lesions may represent a reversible cause of myelopathy . The clinical diagnosis of such unspecific neurologic complex are multifold \& therefore it is not surprising that patients with AVFS \& AVMS make visits to multi disciplinary persons viz orthopaedic surgeons, psychiatrists for erectile dysfunctions, urologists for urinary retention which is misinterpreted as being related to prostate or bladder pathology $y^{3,4}$ The similar situation was observed in the present case who had initially consulted urosurgeon for bladder retention without myelopathic changes \& underwent invasive procedures like cystoscopy, ascending cystourethrography with no avail \& merely resulted in delaying of the diagnosis.

The pathophysiology of symptoms depends on type of AVM. High flow AVMs may lead to ischaemia or haemorrhage. Slow flow may result in venous congestion \& may cause compression of spinal cord $\&$ ischaemia . Most common 4 types of AVMs are based on their location and angioarchitecture, Intradural vs extradural \& or presence or absence of AV Shunts. In 1992 Ansen and Spetzler ${ }^{5}$ classified AVMs into 4 types : Type 1 - Most common type comprising $60 \%$ of spinal AVF/AVM ${ }^{6}$ Often presenting with single A.V. connection within the dura of nerve sheath . Commonly noted in thoracolumbar region ${ }^{7}$. Patient becomes symptomatic because AVF creates venous congestion,hypertension,hypoperfusion,oedema \& hypoxia. Slow flow pathophysiologic feature is responsible for progressive spinal cord ischaemia \& rarely haemorrhage.Elderly male persons are frequently found affected between fifth \& eighth decades of life.

Type 2 (Glomus AVMS) - The entity is analogous to intracranial AVMS.This malformation consists of tightly packed nidus of dysmorphic arteries inside a short segment of cords. The nidus may be completely or partially intramedullary often noted at cervicothoracic junction.It represents $20 \%$ of all spinal vascular malformations.Lesions present in younger patients with acute neurologic deterioration secondary to location which is usually in dorsal cervico medullary region. The reported mortality is $17.6 \%$. After initial haemorrhage , the rebleed is $10 \%$ within $1^{\text {st }}$ month \& $40 \%$ within 1 st year.Vascular steal flow mechanism \& the mass effect may cause myelopathy or radiculopathy. Flow within these lesions is high pressure low flow with rapid filling on angiogram with early venous drainage ${ }^{7}$. The lesions generally present at average age 24 yrs.

Type 3(Juvenile AVMS)-These malformations are arteriovenous abnormalities of spinal cord which are extremely rare.Lesions arise from single or multiple sites thus intradural \& extradural involvement of the cord is seen.Cord tissue is present within the AVM interspaces. The lesions are AVMS having intramedullary nidus which may occupy entire spinal canal at the involved level.

Type 4 Periampullary arteriovenous fistula(AVF)

The lesions are similar to type 1 AVM but are not true AVMS,instead are arteriovenous fistulas.Fistulous connections are intradural but extramedullary without a definable nidus. They maintain high flow lesions with vascular steal/ischaemia,haemorrhage/mass effect as pathophysiologic occurrence.

The endovascular treatment by interventional radiologist for AVM continues to evolve. Treatment options are dictated by the location of the lesion, patients medical condition $\&$ the risk v/s benefit ratio . Advances are probably coming up in catheter technology,image resolution \& embolisation materials. Embolisation with a liquid embolic agent is the first choice treatment for type $2-4$ malformation, where as surgery may be a better option for type 1 malformation ${ }^{8}$.In type 2 AVMS surgery remains the gold standard.However in present case as there was a single feeder \& the location of the feeder was close to foramina magnum endovascular treatment was preferred over surgical intervention.The use of non invasive techniques such as stereolitic spinal radio imaging is presently under evaluation.Patients with intraadural lesions present with mass effect caused by AVM. Enlargement of AVM complexes the surrounding neural tissue, impairing the neurological function.Intradural spinal vascular malformations(Type 2-4) develop during embryogenesis.Such AVM may present with upper or lower extremity involvement,as opposed to patients with dural AVF who typically have only lower limb involvement.Physical examination findings in type of spinal malformation are as follows-

Bruit over spinal cord - Intradural AVM

Hyper reflexia caudal lower lesion,upper motor signs, weakness.

Increased tone - dural AVF and intradural AVM

Saddle sensory disturbance - Dural AVF with gait disturbances.

MRI with MR angiography(MRA)are useful for the initial screening of AVMS.Selective spinal angiography remains the gold standard for definitive diagnose.

Patients who are presenting with bowel or bladder dysfunction and poor antigravity muscle strength in the lower extremities before treatment are noticed to have a limited neurological recovery ${ }^{8}$. Spinal arterio venous malformations-should be included with differential diagnosis of any patient presenting with progressive myelopathy and a cord mass effect recognised on imaging studies. 


\section{Referrences :}

[1]. Rosen blum B , Oldfield EH, Doppman JL, Di Chiro G. Spinal arteriovenous malformations : A complication of dural arterivenous malformations : A comparison of dural arteriovenous fistulas and intradural AVMs in 81 patients . J Neurosurg 1987; 67 : 795-802.

[2]. Barnwell SL, Halbach VV , Dowd CF , Higashida RT , Hieshima GB , Wilson CB . Multiple dural arteriovenous fistulas of craniu m and spine .Am J Neuroradiol 1991;12:441-5.

[3]. Sheikh SI,Busl KM,Ning M, et al.Spinal dural arteriovenous fistula mimicking prostate hyperplasia.J Emerg Med 2008. ( Epub ahead of print )

[4]. Jellema K, Tijssen CC, van Rooij WJ,et al. Spinal dural arteriovenous fistulas: long term follow-up of 44 treated patients.Neurology 2004;62:1839-41

[5]. Anson JA, Spetzler RF. Interventional neuroradiology for spinal pathology . ClinNeurosurg.1992; $39: 388-417$ (Medicine).

[6]. Patsalides A , Santillan A , Knopman J , et al . Endovascular management of spinal dural arteriovenous fistulas . J NeuroIntervent Surg.2010;3(1):80-84.

[7]. Retliff J Connolly $\quad$ E. Spinal Arteriovenous http://www.medschool.lsuhsc.edu/neurosurgery/nervecenter/spavm.html.Updated

[8]. Harrop J S .Vascular malformations of the spinal cord.http:emedicine.medscape.com/article/248456-overview.Updated Aug 17,2011.

\section{Legends to figures:}

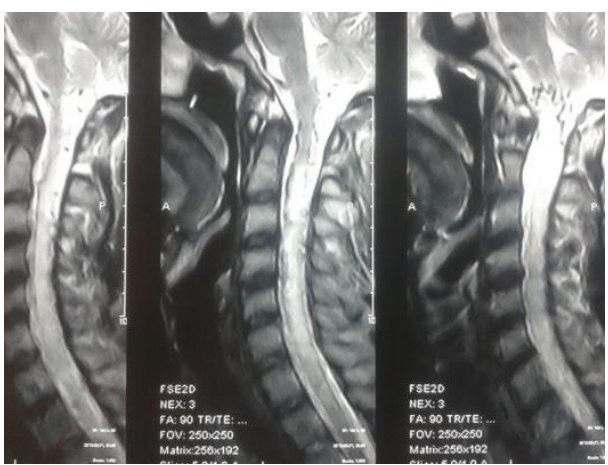

Fig 1 - MRI cervical Spine showing hyperintense signal in spinal cord extending from $C_{2}-C_{7}$ level suggest cord oedema with serpengious flow void .

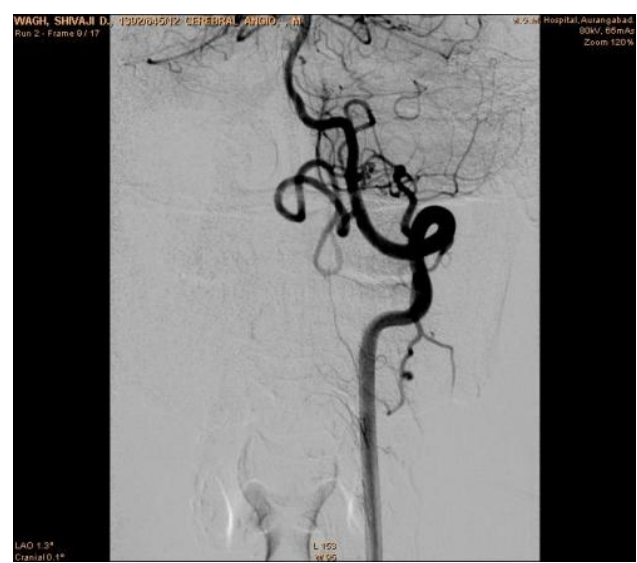

Fig 2 - Left vertebral artery DSA image shows a dilated tortuous vessel from vertebral artery at Foramina Magna.

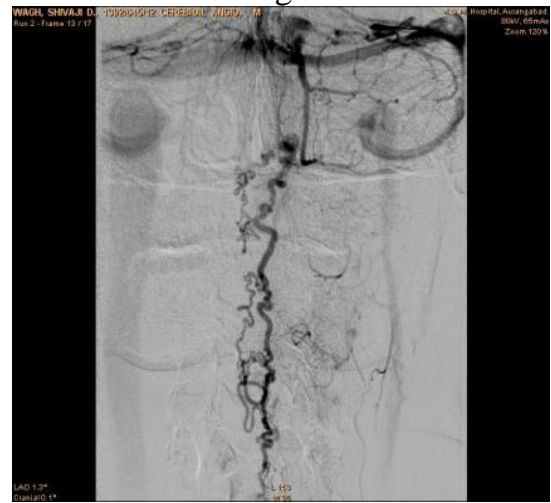

Fig 3 - Left vertebral artery delayed phase DSA image shows AV Malformation(AVF) from left vertebral artery extending to $\mathrm{C} 7$. 


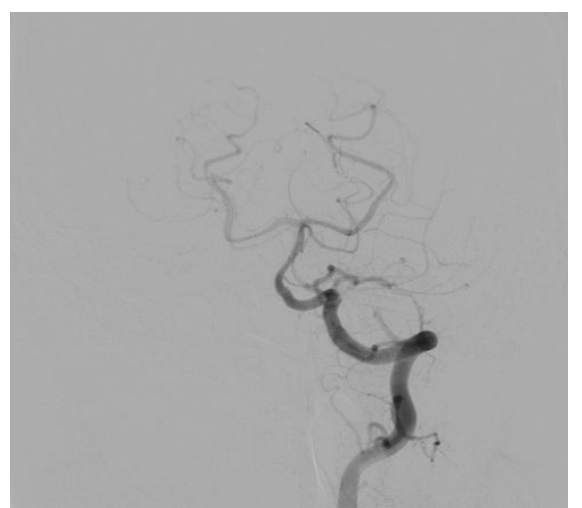

Fig 4 - Post glue embolisation, Left vertebral artery shows complete exclusion of the lesion(AVM).

\section{Acknowledgements :}

The authors remain thankful to Dr Mishrikotkar, Professor \& Head Of Radiology for undertaking imaging studies. 